\title{
УДК 334.723
}

\section{E.D. Schetinina, Yu.N. Bozhkov, S.S. Togba \\ PROBLEMS OF SMALL AND MEDIUM- SIZED ENTERPRISES IN THE REPUBLIC OF CÔTE D'IVOIRE IN THE CONTEXT OF REGIONAL COMPETITIVENESS}

The purpose of the article is to study, generalize and systematize the factors of development of small and medium-sized enterprises in Ivory Coast and identify the relationship between them and the regional competitive strategy and position. This study therefore examines the difficulties and problems of small and medium-sized enterprises in Côte d'Ivoire on the one hand, based on empirical data, and on the other, from the perspective of regional competitiveness, its components and planning. SMEs can and should become drivers of the region's development-with appropriate policies. For example, improved water supply and a welldeveloped licensing policy for production facilities will allow companies in the Ivory Coast to significantly increase their profitability. The deterioration of access to Finance leads to a significant decrease in business sales. In addition, political stability

Keywords: small and medium-sized enterprises (SMEs), problems and obstacles to the functioning of SMEs, model of analysis of small and medium-sized enterprises of the Republic of Ivory Coast, drivers, correlation between restrictions for SMEs and factors of regional competitiveness.

\section{Е.Д. Щетинина ${ }^{1}$, Ю.Н.Божков ${ }^{2}$, С.С.Тогба ${ }^{3}$ \\ ПРОБЛЕМЫ МАЛЫХ И СРЕДНИХ ПРЕД- ПРИЯТИЙ РЕСПУБЛИКИ КОТ-Д'ИВУАРА В КОНТЕКСТЕ РЕГИОНАЛЬНОЙ КОНКУ- РЕНТОСПОСОБНОСТИ}

Целью статьи является изучение, обобщение и систематизация фракторов развития малых и средних предприятий в Котд'Ивуаре и выявление связи между ними и региональной конкурентной стратегией, а также позицией. Поэтому в настоящем исследовании трудности и проблемы малых и средних предприятий в Кот-д'Ивуаре рассматриваются, с одной стороны - на основе данных эмпирики, а с другой - с позиций региональной конкурентоспособности, её составляющих и планирования. МСП могут и должны стать драйверами развития региона - при оказании соответствующей политики. Так, улучшенное водоснабжение и проработанная политика лицензирования производственных помещений позволят компаниям республики Кот-д'Ивуара существенно повысить свою прибыльность. Ухудшение доступа к финансированию влечёт за собой значительное снижение объёма продаж бизнеса. Кроме того, политическая стабильность, реформа налоговой политики и отсутствие коррупции, скорее всего, улучшат финансовое положение компаний и будут способствовать росту продаж бизнеса. Таким образом, все реформы, проводимые правительством Котд'Ивуара для улучшения делового климата, должны учитывать особенности компаний, а также взаимосвязь между различными препятствиями, с которыми они сталкиваются, но главное - они одновременно выступают фракторами конкурентоспособности страны и региона.

Ключевые слова: малые и средние пред-

\footnotetext{
${ }^{1}$ щетинина Е.Д., заведующая кафедрой маркетинга, доктор экономических наук, профессор; Белгородский государственный технологический университет им. В.Г. Шухова, г. Белгород

Schetinina E.D., Head of the Marketing Department, Doctor of Economics, Professor; Belgorod State Technological University named after V.G. Shukhov, Belgorod

E-mail: schetinina@inbox.ru

${ }^{2}$ Божков Ю.Н., доцент кафедры менеджмента и внешнеэкономической деятельности, кандидат экономических наук, доцент; Белгородский государственный технологический университете им. В.Г. Шухова, г. Белгород

Bozhkov Yu.N., Associate Professor of the Department of Management and Foreign Economic Activity, PhD in Economics, Associate Professor; Belgorod State Technological University named after V.G. Shukhov, Belgorod

E-mail: belgo@mail.ru

${ }^{3}$ Тогба С.С., аспирант; Белгородский государственный технологический университет им. В.Г. Шухова, г. Белгород

Togba S.S., Postgraduate; Belgorod State Technological University named after V.G. Shukhov, Belgorod

E-mail: stephanietogba@gmail.com
} 
приятия (МСП), проблемы и препятствия функционирования МСП, модель анализа малых и средних предприятий республики Кот-д'Ивуар, драйверы, корреляция между ограничениями для МСП и факторами региональной конкурентоспособности.

DOI: 10.36807/2411-7269-2021-1-24-107-114

Введение. Малые и средние предприятия являются ключевыми фракторами экономического развития развивающихся стран. Крайне важно определить основные препятствия, с которыми они сталкиваются. С целью стимулирования своего частного сектора, Кот-д'Ивуар выбрал экономическую либерализацию, чтобы привлечь в страну иностранные инвестиции. В основе этого политического выбора лежит особое значение, придаваемое развитию частного сектора. Кот-д'Ивуар рассматривал частный сектор как движущую силу экономического роста, инструмент экономической интеграции и стратегию сокращения масштабов нищеты путём создания рабочих мест и получения доходов. В рамках документа о стратегии сокращения масштабов нищеты 2012 г. [12], власти Котд'Ивуара подчеркнули важность развития частного сектора как двигателя роста. Для достижения этой цели важно, чтобы частный сектор функционировал в оптимальных условиях. Это среда, в которой инвестиционные операции и вытекающая из них деятельность защищены и организованы надёжными законами и правилами. Кроме того, существует оптимальная среда, которая управляется надёжными структурами и финансируется динамичными институтами, а также эфффективным фринансовым рынком. Причина в том, что в системе экономического либерализма все заинтересованные стороны основывают большинство своих решений на сигналах, которые они получают от правительства и рынка.

К сожалению, частный сектор Кот-д'Ивуара остаётся запятнанным последовательными повторяющимися кризисами, которые ставят его в действительно тяжёлое положение. Экономика Кот-д'Ивуара пострадала от социально-политических кризисов 1999 и 2012 гг. Эти кризисы начались с военного переворота, закончились послевыборным кризисом 2011 г. и в целом повлияли на предоставление и качество основных услуг. Результаты этих кризисов не обошлись без последствий для экономики и малого и среднего бизнеса. Согласно отчёту Всемирного банка по благоприятности условий ведения бизнеса по состоянию на май 2019 г., Кот-д'Ивуар занимает 110 место из 190 мировых экономик.

Кроме того, по данным Министерства промышленности и развития частного сектора, социально-политические кризисы 1999 и 2011 гг. имели четыре основных последствия. Во-первых, это исчезновение половины малых и средних предприятий (МСП)/малых и средних промышленных предприятий (МСПП) и уничтожение 78 крупных компаний. Во-вторых, было частичное или полное закрытие промышленных предприятий в районах, пострадавших от кризиса, особенно в центральной, северной и западной зоне. В-третьих, произошло перемещение ряда компаний в другие страны субрегиона, в общей сложности 226 компаний с 1999 по 2007 гг. В-четвёртых, это потеря многих рабочих мест в формальном частном секторе (более 500000 рабочих мест по данным Торговопромышленной палаты Кот-д'Ивуара) и доли рынка на региональном и международном уровнях [25]. Таким образом, эти кризисы серьёзно повлияли на рост и сбалансированность национальной экономики, поставив частный сектор в очень рискованную и неблагоприятную деловую среду [8].

Политическая поддержка частного сектора, осуществляемая как правительствами, так и международными и частными заинтересованными сторонами, в целом, сосредоточена на совершенствовании нормативной базы, финансировании, поддержке профессиональной подготовки и различных налоговых стимулах для поддержания и развития предпринимательской деятельности. Так, в последние годы в Кот-д'Ивуаре было зафиксировано значительное число стимулов и специальных дискреционных налоговых льгот, направленных на поддержку частного сектора и постконфликтное восстановление [15], [11]. Однако результаты этой политики остаются неоднозначными [21]. Ивуарийские компании неэфрфективны, и сегодня страна производит добавленную стоимость на душу населения значительно ниже уровня, который был 20-25 лет назад. После различных реформ, предложенных правительством Кот-д'Ивуара, представляется более чем необхо- 
димым рассмотреть данные за последнее время и в микроэкономическом контексте, чтобы понять трудности, с которыми сталкиваются компании, работающие в Кот-д'Ивуаре.

Методы исследования. Исследование базировалось на общенаучных методах познания (диалектике, индукции и дедукции, анализе и синтезе) сравнительном и структурно-логическом анализе, эмпирическом обобщении, табличных и графических приёмах визуализации статистических и расчётных данных.

Результаты исследования. До сих пор исследования по МСП в Кот-д'Ивуаре были весьма скудными. В предыдущих исследованиях [18], [23] основное внимание уделялось одному конкретному ограничению. Данная работа распространяет свой анализ на различные типы ограничений, о которых сообщают МСП. Интерес этого исследования заключается в том, что оно может дать ответ, который может быть использован для реформирования деловой среды в ответ на императив посткризисной политики экономического восстановления в Кот-д'Ивуаре.

Анализ восприятия ограничений в соответствии с характеристиками компаний показал, что восприятие уровня ограничений является функцией характеристик бизнеса, и поэтому любая политическая реформа должна основываться на этих характеристиках бизнеса. Одним из важных результатов этого исследования, основанного на работе Т. Бек и др. [9], является подход, который заключается в том, что доступ к финансированию считается наиболее важным ограничением, негативно влияющим на рост продаж компании. Ухудшение на 1 процентный пункт условий доступа к финансированию сопровождается резким падением на 148 процентных пунктов темпов роста бизнеса. Кроме того, мы находим, что улучшение условий водоснабжения и разрешений на строительство приводит к улучшению роста бизнеса.

Ряд авторов изучили ограничения, с которыми сталкиваются МСП [9], [11], [12]. В то время как прошлые исследования были сосредоточены в основном на финансовых показателях, в более новых работах был сделан акцент на широком спектре ограничений, предоставляемых бизнес-исследованиями. Согласно исследованию делового климата [24], [18], ограничения, с которыми сталкиваются предприятия, можно разделить на несколько категорий, а именно:

- $\quad$ финансовые: стоимость кредита, доступ к кредиту;

- $\quad$ эффрективность судебной системы: безопасность, защита прав собственности, эффективное администрирование правосудия;

- налоги и регулирование: налоги, регулирование, недобросовестная конкуренция;

- инфраструктурные проблемы: качество и практичность дорог, электричества, водоснабжения, телефонной связи, почтовой связи;

- $\quad$ коррупция: качество взаимоотношений с государственными службами;

- более широкая макроэкономическая среда: преступность, политическая нестабильность, колебания валютных курсов, инфляция.

Многие эмпирические исследования выявили ограниченный доступ к финансированию и неэффективность судебной системы в качестве основных препятствий для роста бизнеса. Некоторые авторы, такие как Ла Порта, Лопес-де-Силанес, Шлейфери Вишни [19], утверждают, что различия между правовыми системами и финансовыми системами разных стран могут объяснить различия в эффективности бизнеса в мире.

Несколько исследований [14], [17] описали, как неблагоприятный деловой климат негативно влияет на функционирование и рост предприятий. Однако многие из них ограничены данными из одной страны и сосредоточены, как правило, на одном ограничении.

Л. Славугени, М. Гойдхойс на основе данных о предприятиях в Кот-д'Ивуаре показывают, что неудовлетворительная финансовая инфраструктура негативно сказывается на развитии малого бизнеса [23]. Р. Левин подчёркивает важность финансового развития для экономического роста за счёт повышения доступности кредитов [20]. Кроме того, другие исследователи уделяют особое внимание коррупции по сравнению с уплатой налогов. Одна из первых работ в этой области была предложена Шлейфером и Вишни [22]. Они утверждают, что коррупция может быть более разрушительной, чем уплата налогов из-за неопределённости и секретности. Хотя эти исследования помогли улучшить понимание влияния деловой среды на развитие бизнеса между странами, но они изучают конкретный аспект ограничений и имеют недостаточные рекомендации. Другие авторы анализируют ограничения в гораздо более сложной среде. К. Куад показал, что трудности, тормозящие развитие фирм Кот-д'Ивуара, состоят из трёх частей: 1) фринансовых ограничений; 2) низкого уровня социальной эфффективности фракторов производства (неблагоприятных географических фракторов или недостаточного инвестирования дополнительных факторов, 
таких как человеческий капитал и инфрраструктура); 3) малого объёма ассигнований (высокие макро- и микрориски, неэффективное налогообложение на право собственности и обеспечение исполнения контрактов, малое количество или отсутствие продуктовых инноваций, значительные экстерналии). Кроме того, другие исследования, помимо тех, что были проведены в Кот-д'Ивуаре, выявили в качестве основных ограничений деловой активности отсутствие фринансирования и отсутствие корпоративной сети. Херрингтон и его коллеги обнаружили, что отсутствие образования (в том числе в области человеческого капитала) и профессиональной подготовки является наиболее важной причиной неудач для новых МСП в Южной Африке [16].

Переходя к рассмотрению проблем функционирования МСП в данной статье, стоит отметить, что одним из основных источников исследования делового климата в Котд'Ивуаре является работа "ClimatdesaffairesCôted'Ivoire" автора К.Н. Kouadio и его коллег [18]. Этот опрос выявил основные препятствия на пути деятельности и роста фрирм в 6 регионах страны. Он содержит ряд важных вопросов о характере серьёзности различных препятствий. В частности, бизнес-лидерам предлагается высказать своё мнение о препятствиях, связанных с финансами, коррупцией, налогами и регулированием, инфрраструктурой, правосудием, преступностью, политической нестабильностью и макроэкономической средой. Цель этого исследования состояла в том, чтобы: 1) представить статистические показатели деловой среды; 2) понять фракторы, сдерживающие рост частного сектора; 3) стимулировать политические реформы для улучшения делового климата.

Опрос охватил 727 компаний, расположенных в отдельных городах, включая 414 офрициальных и 313 неофициальных предприятий (Табл. 1).

Таблица 1 - Данные по опрошенным предприятиям

\begin{tabular}{|c|c|c|c|c|c|}
\hline & $\begin{array}{l}\text { Офицци- } \\
\text { альные }\end{array}$ & $\begin{array}{l}\text { Неофиици- } \\
\text { альные }\end{array}$ & & $\begin{array}{l}\text { Офрици- } \\
\text { альные }\end{array}$ & Неофиции-альные \\
\hline \multicolumn{3}{|l|}{ Города (в \%) } & \multicolumn{3}{|l|}{ Возраст (в \%) } \\
\hline Абиджан & 10,1 & 14,4 & 1 до 5 лет & 36 & 38 \\
\hline Сан-Педро & 8 & 3,8 & 6 до 10 лет & 20,5 & 21,1 \\
\hline Абенгуру & 21 & 20,1 & 11 до 15 лет & 12,1 & 14,4 \\
\hline Буак & 23 & 16,6 & 16 до 20 лет & 8,5 & 11,2 \\
\hline Далоа & 20,5 & 20,8 & 21 до 35 лет & 6 & 4,8 \\
\hline \multirow[t]{2}{*}{ Kорого } & 17,4 & 24,3 & 26 до 30 лет & 5,3 & 3,2 \\
\hline & & & Более 30 лет & 11,6 & 7,3 \\
\hline \multicolumn{3}{|c|}{ Статус предприятия (в \%) } & & & \\
\hline $\begin{array}{l}\text { Анонимное } \\
\text { общество }\end{array}$ & 12,3 & 0,3 & \multicolumn{3}{|c|}{ Размер предприятия (в \%) } \\
\hline $\begin{array}{l}\text { Общество с } \\
\text { ограниченной } \\
\text { ответствен- } \\
\text { ностью } \\
\end{array}$ & 21,3 & - & Микро & 46,2 & 77 \\
\hline $\begin{array}{l}\text { Индивиду- } \\
\text { альный биз- } \\
\text { нес }\end{array}$ & 58,5 & 93 & Малые & 37,9 & 23 \\
\hline Партнёрство & 3,4 & 3,2 & Средние & 11,8 & - \\
\hline $\begin{array}{l}\text { Коммандит- } \\
\text { ное товари- } \\
\text { щество }\end{array}$ & 0,5 & 0,3 & Крупные & 4,1 & - \\
\hline \multirow[t]{4}{*}{ Другие } & 4,1 & 3,2 & & & \\
\hline & & & \multicolumn{3}{|c|}{ Сектор экономики (в \%) } \\
\hline & & & Промышлен-ность & 18,6 & 49,2 \\
\hline & & & Сервис & 81,4 & 50,8 \\
\hline
\end{tabular}

Источник: взято авторами из [18].

Выборка опроса была разделена по следующим пунктам: промышленность, размер и регион. Из выборки были исключены правительственные департаменты, военные базы, полицейские префектуры, школы, университеты, центры общественного здравоохранения или другие символы государственных структур.

Анкета содержала несколько тематических структур, характеристики фрирмы (возраст фрирмы, размер, юридический статус и т.д.), доступ к инфраструктуре (электроэнергия, транспорт, водоснабжение), отношения с государством (регуляторные, административные обязанности, коррупция, разрешения или лицензии), наёмные работники (количество постоянных и временных работников), показатели деятельности фрирмы (загрузка производственных мощностей, продажи, экспорт), доступ к фринансам (банковский счёт, источник финансирования и т.д.) и основные барьеры. 
В Табл. 2 обобщён уровень барьеров, с которыми сталкиваются руководители предприятий, которые приняли участие в опросе. В этой таблице также показано, как изменялся процент того или иного ограничения в зависимости от размера компании.

Таблица 2 - Статистика встречающихся препятствий в процессе функционирования предприятий в зависимости от размера компании

\begin{tabular}{|c|c|c|c|c|c|c|}
\hline \multirow{2}{*}{$\begin{array}{c}\text { Существую- } \\
\text { щие препят- } \\
\text { ствия }\end{array}$} & \multirow{2}{*}{ Уровень барьеров } & \multicolumn{5}{|c|}{ Размер предприятий } \\
\hline & & Микро & Малые & Средние & Крупные & Всего \\
\hline \multirow{4}{*}{$\begin{array}{l}\text { Электроэнер- } \\
\text { гия }\end{array}$} & Нет & 32,41 & 24,45 & 20,41 & 11,76 & 28,61 \\
\hline & Умеренное & 11,81 & 10,92 & 14,29 & 29,41 & 12,1 \\
\hline & Существенное & 19,68 & 29,69 & 32,65 & 11,76 & 23,52 \\
\hline & Сильное & 36,11 & 34,93 & 32,65 & 47,06 & 35,76 \\
\hline \multirow{4}{*}{$\begin{array}{l}\text { Водоснабже- } \\
\text { ние }\end{array}$} & Нет & 74,54 & 54,59 & 46,94 & 47,06 & 65,75 \\
\hline & Умеренное & 7,64 & 11,79 & 26,53 & 17,65 & 10,45 \\
\hline & Существенное & 9,26 & 15,72 & 14,29 & 11,76 & 11,69 \\
\hline & Сильное & 8,56 & 17,9 & 12,24 & 23,53 & 12,1 \\
\hline \multirow{4}{*}{ Конкуренция } & Нет & 35,19 & 34,06 & 38,78 & 52,94 & 35,49 \\
\hline & Умеренное & 18,06 & 17,03 & 8,16 & 0 & 16,64 \\
\hline & Существенное & 22,69 & 26,64 & 26,53 & 23,53 & 24,21 \\
\hline & Сильное & 24,07 & 22,27 & 26,53 & 23,53 & 23,66 \\
\hline \multirow{4}{*}{$\begin{array}{l}\text { Преступления, } \\
\text { грабежи }\end{array}$} & Нет & 41,2 & 41,48 & 44,9 & 47,06 & 41,68 \\
\hline & Умеренное & 12,27 & 13,54 & 12,24 & 11,76 & 12,65 \\
\hline & Существенное & 22,69 & 21,83 & 18,37 & 23,53 & 22,15 \\
\hline & Сильное & 23,84 & 23,14 & 24,49 & 17,65 & 23,52 \\
\hline \multirow{4}{*}{$\begin{array}{l}\text { Доступ к фи- } \\
\text { нансированию }\end{array}$} & Нет & 22,22 & 27,51 & 36,73 & 35,29 & 25,17 \\
\hline & Умеренное & 13,66 & 14,85 & 14,29 & 29,41 & 14,44 \\
\hline & Существенное & 27,31 & 29,26 & 14,29 & 23,53 & 26,96 \\
\hline & Сильное & 36,81 & 28,38 & 34,69 & 11,76 & 33,43 \\
\hline \multirow{4}{*}{$\begin{array}{l}\text { Налоговая } \\
\text { ставка }\end{array}$} & Нет & 29,86 & 29,26 & 30,61 & 29,41 & 29,71 \\
\hline & Умеренное & 21,99 & 19,21 & 24,49 & 35,29 & 21,6 \\
\hline & Существенное & 27,08 & 30,13 & 28,57 & 23,53 & 28,06 \\
\hline & Сильное & 21,06 & 21,4 & 16,33 & 11,76 & 20,63 \\
\hline \multirow{4}{*}{$\begin{array}{l}\text { Политическая } \\
\text { нестабиль- } \\
\text { ность }\end{array}$} & Нет & 6,02 & 7,42 & 2,04 & 5,88 & 6,19 \\
\hline & Умеренное & 5,32 & 3,06 & 4,08 & 5,88 & 4,54 \\
\hline & Существенное & 20,14 & 22,71 & 32,65 & 11,76 & 21,6 \\
\hline & Сильное & 68,52 & 66,81 & 61,22 & 76,47 & 67,68 \\
\hline \multirow{4}{*}{ Коррупция } & Нет & 45,37 & 48,03 & 36,73 & 47,06 & 45,67 \\
\hline & Умеренное & 13,66 & 9,17 & 12,24 & 5,88 & 11,97 \\
\hline & Существенное & 20,14 & 22,27 & 20,41 & 5,88 & 20,5 \\
\hline & Сильное & 20,83 & 20,52 & 30,61 & 41,18 & 21,87 \\
\hline
\end{tabular}

Источник: взято из [18]

Из данного анализа видно, что три препятствия считаются серьёзными для функционирования компании. К ним относятся доступ к электричеству, доступ к финансированию и проблема политической нестабильности. Действительно, почти 59 \% руководителей различных компаний сообщили о ведении бизнеса в условиях ограничения на электроэнергию, в то время как 60 \% считают, что фринансовые ограничения негативно сказываются на фрункционировании бизнеса. Примерно 80 \% тех, кто считает, что политическая нестабильность не позволяет развиваться их бизнесу. Результаты опроса также показывают, что ни земельные, ни транспортные, ни административные сборы, ни снабжение питьевой водой не рассматриваются в качестве основных препятствий для развития компании. Кроме того, хотя они и не заняли первое место в рейтинге ограничений, они более чем на 40 \% заявили, что недобросовестная конкуренция (47,87 \%), преступления (45\%), налоговые ставки (48 \%), коррупция (42 \%) представляют серьёзные препятствия для роста их бизнеса.

Однако в Табл. 2 показаны различия в восприятии в зависимости от размера компании. Из Табл. 2 видно, что микропредприятия в основном (64 \%) рассматривают проблему доступа к финансированию как серьёзное препятствие для роста своего бизнеса. За ними следуют малые (57 \%), средние (49 \%) и крупные компании (35\%). Что касается ограничений на электроэнергию, то их восприятие варьируется в зависимости от размера компании. Самые многочисленные малые и средние предприятия считают, что эти барьеры являются сдерживающим фрактором для их бизнеса. Они составляют около 65 \% всех малых и средних предприятий. 
В работе "ClimatdesaffairesCôted'Ivoire" автора K.H. Kouadio и его коллег представлена матрица корреляции между ограничениями. Препятствия, о которых сообщают фрирмы, важны, но корреляции остаются довольно низкими, ниже 0,5. Корреляция между политической нестабильностью и преступностью и коррупцией остаётся относительно высокой. Этот результат свидетельствует о том, что экономическая среда, характеризующаяся политической нестабильностью, неизбежно влияет на коррупцию. Важно также отметить, что корреляция между доступом к фринансированию и другими барьерами относительно высока, что подчёркивает, что любая политика реформ должна учитывать возможные взаимосвязи между этими ограничениями [18].

Заключение. Предварительные результаты показали, что наиболее тревожными препятствиями для предпринимателей являются ограничения доступа к фринансированию, политическая нестабильность и нехватка электроэнергии. Эти же фракторы, особенно первые два, выступают ключевыми для обеспечения конкурентоспособности страны в целом.

Более того, анализ восприятия ограничений в соответствии с характеристиками компании показал, что восприятие уровня ограничений зависит от характеристик компании. Таким образом, любая политика реформ должна учитывать особенности предприятий, тем более что МСП очень неоднородны. Также необходимо отметить, что по мере решения проблем, связанных с доступом к финансированию, правительство должно сосредоточиться на соинвестировании в повышение квалификации сотрудников МСП и развитие человеческого капитала.

Таким образом, исходя из анализа научных работ, официальных данных государственных структур республики Кот-д'Ивуар, можно утверждать, что правительству следует решать эти проблемы в интеграции с разработкой стратегии укрепления конкурентной позиции страны и даже региона. Коллаборация, объединение с прочими участниками новые формы решения задач экономики Кот-д'Ивуара. В общую стратегию должны быть включены задачи, в том числе связанные с ограничениями и препятствиями, встречающимися перед МСП, и способы их решения. Очевидно, что целый ряд важных экономических и социальных стратегий, таких как макроэкономическая политика, инфраструктура или социальная защита, окажут существенное влияние на МСП, ставя целью сделать МСП драйверами внутреннего роста и конкурентной устойчивости. Три генеральных элемента политики республики в отношении МСП и одновременно роста конкурентоспособности заключаются в: 1) устранении провалов рынка, характерных для данного сегмента предприятий за счёт ресурсных и финансовых преференций; 2) поощрении особого вклада МСП в экономику республики и рост конкурентоспособности страны; 3) информационно-маркетинговая поддержка МСП.

\section{Список использованных источников}

1. Адамчук А.М. Причины, сдерживающие развитие промышленных малых предприятий / А.М. Адамчук, Д.Е. Баркин // Вестник БГТУ им. В.Г. Шухова. - 2016. - № 1. С. $165-168$.

2. Бендерская О.Б. Динамика развития малого бизнеса // Белгородский экономический вестник. - 2018. - № 4. - С. 16-26.

3. Багаева М.В. Сколько стоит малый бизнес? Выбор системы налогообложения / М.В. Багаева. - М.: Феникс, 2016. - 256 с.

4. Браверман А.А. Маркетинг для прибыльности среднего бизнеса: моногр. / А.А. Браверман. - М.: Экономика, 2015. - 211 с.

5. Джобава Н.А. Государственное регулирование малого предпринимательства / Н.А. Джобава. - М.: Издательский дом Санкт-Петербургского государственного университета, Издательство юридического факультета Санкт-Петербургского государственного университета, 2018. - 112 с.

6. Дэвид Д. Предпринимательство и малые фирмы / Дикинс Дэвид. - М.: Институт прикладной психологии "Гуманитарный центр", 2017. - 618 с.

$995 \mathrm{c}$.

7. Орлов А.В. Менеджмент в малом бизнесе / А.В. Орлов. - Москва: ИЛ, 2018. -

8. Всемирный банк "Рейтинг стран мира" [Электронный ресурс]. - Режим доступа: https://russian.doingbusiness.org/ru/rankings (16.07.2020).

9. Beck T., Demirgüç-Kunt A., Maksimovic V. Financial and Legal Constraints to Firm Growth: Does Firm Size Matter / T. Beck, A. Demirgüç-Kunt, V. Maksimovic // Journal of Finance, 2005 - № 60(1). - PP. 137-177. 
10. CEPICI. Retrospective doing business 2015 / CEPICI // Newsletter. -2015. - November. - PP. 1-8.

11. Dollar D., Hallward-Driemeier M., Mengistae T. Investment Climate and Firm Performance in Developing Economies / D. Dollar, M. Hallward-Driemeier, T. Mengistae // Economic Development and Cultural Change, 2005. -№ 54(1). - PP. 1-21.

12. Dollar D., Hallward-Driemeier M., Mengistae T. Investment climate and international integration / D. Dollar, M. Hallward-Driemeier, T. Mengistae // World Development. - 2006. № 34(9). - PP. 1498-1516.

13. DSRP. Stratégie de Relance du Développementet de Réduction de la Pauvreté / DSRP // République de Côte d'Ivoire, 2012. - 180 p.

14. Fjose S., Grünfeld L.A., Green C. SMEs and growth in Sub-Saharan Africa: Identifying SME roles and obstacles to SME growth / S. Fjose, L.A. Grünfeld, C. Green // MENONpublication, 2010. - № 14. -28 p.

15. GCCl. Note sur l'environnement des affaires en Côte d'Ivoire, secrétariat technique du GroupeConsultatif Plan National de Développement 2016-2020 / GCCl // Côte d'Ivoire, 2016.

16. Herrington M., Kew J., Kew P. Global Entrepreneurship Monitor / M. Herrington, J. Kew, P. Kew // South African Report, 2009. - 24 p. [Electronic resource]. - URL: https://link.springer.com/content/pdf/10.1186/s40497-019-0183-1.pdf. (18.07.2020).

17. ILO. Small and medium-sized enterprises and decent and productive employment creation, Report IV / ILO // International Labour Conference, 104th Session, Geneva, 2015. - 84 p.

18. Kouadio K. Climat des affaires Côte d'Ivoire 2012: Méthodologie et Outils de Collecte, Rapport d'étude / K. Kouadio, N. Soro, N. Gbenro, D. Kanga // ENSEA, 2013.

19. La Porta R. Law and finance / R. La Porta, F. Lopez-de-Silanes, A. Shleifer, R.W. Vishny // Journal of Political Economy, 1998. - № 106. - PP. 1113-1155.

20. Levine R. Finance and Growth: Theory and Evidence forthcoming in Philippe Aghion and Steven Durlauf / R. Levine // Handbook of Economic Growth. - The Netherlands: Elsevier Science, 2005. - PP. 865-934.

21. ONUDI. Nouvelle politiquelndustrielle de la Côte d'Ivoire / ONUDI // Vienne (Autriche), - 2012. - $130 \mathrm{p}$.

22. Shleifer A., Vishny W. Robert. Corruption / A. Shleifer, W. RobertVishny // The Quarterly Journal of Economics, 1993. - Vol. 108, № 3. - PP. 599-617.

23. Sleuwaegen L., Goedhuys M. Growth of firms in developing countries, evidence from Cote d'Ivoire / L. Sleuwaegen, M. Goedhuys // Journal of Development Economics, 2002. - № 68(1). - PP. 117-135.

24. WBES. Enterprise Surveys: Côte d'Ivoire Country Profile 2009 / WBES // IFC, The World Bank Group. 2010. - 15 p.

25. Official website of the chamber of Commerce and industry of Côte d'Ivoire. [Electronic resource]. - URL: http://www.cci.ci/3.0/ (25.07.2020).

\section{References}

1. Adamchuk A.M., Barkin D.E. (2016). Prichiny, sderzhivayushchierazvitiepromyshlennykhmalykhpredpriyatiy [The Causes hindering the development of small industrial enterprises]. Vestnik BGTU im. V.G. Shukhov [Vestnik BGTU im. V.G. Shukhova]. 1, 165-168.

2. Benderskaya O.B. (2018). Dinamikarazvitiyamalogobiznesa [Dynamics of small business development]. Belgorodskiyekonomicheskiyvestnik [Economic Bulletin of Belgorod]. 4, 16-26.

3. Bagaeva M.V. (2016). Skol'kostoitmalyybiznes? Vyborsistemynalogooblozheniya [How much is a small business? Choosing a tax system]. M.: Feniks [Moscow: Fenix].

4. Braverman A.A. (2015). Marketingdlyapribyl'nostisrednegobiznesa: monogr. [Marketing for the profitability of medium-sized businesses: Monogr.]. M.: Ekonomika [Moscow: Ekonomika].

5. Jobava N.A. (2018). Gosudarstvennoeregulirovaniemalogopredprinimatel'stva
[State regulation of small business]. M.: Izdatel'skiydom SanktPeterburgskogogosudarstvennogouniversiteta, Izdatel'stvoyuridicheskogofakul'teta SanktPeterburgskogogosudarstvennogouniversiteta. [M.: Publishing house of the Saint Petersburg state University, Publishing house of the law faculty of the Saint Petersburg state University]. 
6. David Deakins. (2017). Predprinimatel'stvoimalyefirmy [Entrepreneurship and small firms]. M.: Institutprikladnoypsikhologii "Gumanitarnyytsentr". [M.: Institute of applied psychology "Humanitarian center"].

7. Orlov A.V. (2018). Menedzhment $v$ malombiznese [Management in small business]. Moscow: IL [Moscow: IL].

8. World Bank "Rating of countries of the world". Available: https://russian.doingbusiness.org/ru/rankings.

9. Beck T., Demirgüç-Kunt A. \& Maksimovic V., (2005). Financial and Legal Constraints to Firm Growth: Does Firm Size Matter. Journal of Finance 60(1), 137-177.

10. CEPICI (2015). Retrospective doing business 2015, newsletter novembre 2015.

11. Dollar D., Hallward-Driemeier M. \& Mengistae T., (2005). Investment Climate and Firm Performance in Developing Economies, Economic Development and Cultural Change 54 (1), 1- 21.

12. Dollar D., Hallward-Driemeier M. \& Mengistae T., (2006). Investment climate and international integration, World Development, 34(9), 1498-1516, September.

13. DSRP (2012). Stratégie de Relance du Développementet de Réduction de la Pauvreté, République de Côte d'Ivoire.

14. Fjose S., Grünfeld L.A. \& Green C. (2010). SMEs and growth in Sub-Saharan Africa: Identifying SME roles and obstacles to SME growth, MENON-publication no. 14/2010.

15. GCCI (2016). Note sur l'environnement des affaires encôted'ivoire, secrétariat technique du GroupeConsultatif Plan National de Développement 2016-2020, Côte d'Ivoire.

16. Herrington M, Kew J, Kew P (2009). Global Entrepreneurship Monitor, South African Report. [Online]. Available: http://www.gbs.nct.ac.za/gbswebb/userfiles/gemsouthafrica2000pdf.

17. ILO (2015). Small and medium-sized enterprises and decent and productive employment creation, Report IV, International Labour Conference, 104th Session, Geneva, 2015.

18. Kouadio K. H., Soro N., Gbenro N. \& Kanga D. (2013). Climat des affaires Côte d'Ivoire 2012: Méthodologie et Outils de Collecte, Rapport d'étude, ENSEA.

19. La Porta R., Lopez-de-Silanes F., Shleifer A., Vishny R.W., (1998). Law and finance. Journal of Political Economy 106, 1113-1155.

20. Levine R., (2005). Finance and Growth: Theory and Evidence forthcoming in Philippe Aghion and Steven Durlauf, eds. Handbook of Economic Growth. The Netherlands: Elsevier Science.

21. Ministère de l'Economieet des Finances (2010). Annexe fiscal 2010, Abidjan Côte d'Ivoire ONUDI (2012). Nouvelle politiquelndustrielle de la Côte d'Ivoire, Vienne (Autriche).

22. Shleifer, Vishny (1993). Corruption. The Quarterly Journal of Economics, Vol. 108, No. 3. (Aug., 1993), pp. 599-617.

23. Sleuwaegen L. \& Goedhuys M., (2002). Growth of firms in developing countries, evidence from Cote d'Ivoire, Journal of Development Economics, 68 (1), 117-135.

24. WBES (2010). Enterprise Surveys: CÔTE D'IVOIRE Country Profile 2009, IFC, The World Bank Group.

25. Official website of the chamber of Commerce and industry of Côte d'Ivoire [Online]. Available: http://www.cci.ci/3.0/ (25.07.2020).

Работа содержит результаты НИР "Управление привлекательностью региона на основе маркетинговых технологий" и фринансируется в рамках мероприятий Программы развития опорного университета на базе БГТУ им. В.Г. Шухова до 2021 года. 